\title{
Cross-linking mass spectrometry reveals structural insights of the glutamine synthetase from Leishmania braziliensis
}

\author{
Jhenifer Yonara de Lima', Marlon Dias Mariano Santos' ${ }^{1}$, Mario Tyago Murakami², \\ Paulo Costa Carvalho', Tatiana de Arruda Campos Brasil de Souza ${ }^{1 /+}$ \\ ${ }^{1}$ Fundação Oswaldo Cruz-Fiocruz, Instituto Carlos Chagas, Laboratório de Proteômica Estrutural e Computacional, Curitiba, PR, Brasil \\ ${ }^{2}$ Centro Nacional de Pesquisa em Energia e Materiais, Laboratório Nacional de Biorrenováveis, Campinas, SP, Brasil
}

BACKGROUND Leishmaniasis is a neglected tropical disease caused by the parasite Leishmania braziliensis, commonly found in Brazil and associated with cutaneous and visceral forms of this disease. Like other organisms, L. braziliensis has an enzyme called glutamine synthetase (LbGS) that acts on the synthesis of glutamine from glutamate. This enzyme plays an essential role in the metabolism of these parasites and can be a potential therapeutic target for treating this disease.

OBJECTIVES Investigate LbGS structure and generate structural models of the protein.

METHODS We use the method of crosslinking mass spectrometry (XLMS) and generate structural models in silico using I-TASSER.

FINDINGS 42 XLs peptides were identified, of which 37 are explained in a monomeric model with the other five indicating LbGS dimerization and pentamers interaction region. The comparison of 3D models generated in the presence and absence of XLMS restrictions probed the benefits of modeling with XLMS highlighting the inappropriate folding due to the absence of spatial restrictions.

MAIN CONCLUSIONS In conclusion, we disclose the conservation of the active site and interface regions, but also unique features of LbGS showing the potential of XLMS to probe structural information and explore new drugs.

Key words: structural proteomics - protein - glutamine synthetase - Leishmania braziliensis

According to the Centers for Disease Control and Prevention (CDC), leishmaniasis comprises a group of neglected tropical diseases caused by parasites of the genus Leishmania. This disease presents itself in three forms: cutaneous (CL), mucous (ML), and visceral (VL). Cutaneous leishmaniasis can cause substantial morbidity, while visceral leishmaniasis can be fatal. ${ }^{(1,2)}$ In Brazil, data from 2019 show 2,529 new cases of VL, with 1.2 cases per 100,000 inhabitants and a mortality rate of $9 \%$. For CL, 15,484 new cases were confirmed ( 7.37 cases per 100,000 inhabitants), of which $67.1 \%$ had clinical cure $1.9 \%$ abandoned treatment and 19 deaths. ${ }^{(3)}$ There are at least eight species of Leishmania that can infect humans and lead to the development of parasitosis in Brazil: L. (V.) braziliensis, L. guyanensis, L. (L.) amazonensis, L. (L.) infantum (syn. chagasi) $L$. (V.) lainsoni, $L(V$.$) naiffi, L$. (V.) shawi and $L$. (V.) lindenbergi. ${ }^{(4)}$ Considering that Leishmaniasis is found on every continent except Australia, Pacific Islands and Antarctica, and there are about 90 countries classified as endemic, WHO estimates approximately 1.2 million new cases of cutaneous leishmaniasis per year. ${ }^{(2)}$ For visceral leishmaniasis, it is estimated that the new incidences are currently below 100,000 per year. ${ }^{(2)}$

doi: 10.1590/0074-02760210209

+ Corresponding author: tatiana.brasil@fiocruz.br

(D) https://orcid.org/0000-0002-1850-6080

Received 14 June 2021

Accepted 26 October 2021
The enzyme glutamine synthetase (GS) is essential in nitrogen metabolism, being responsible for the catalysis of glutamine from ATP, glutamate, and ammonia; this process occurs in two stages, starting with the activation of an intermediate gamma-glutamyl phosphate ( $\gamma-\mathrm{G}-\mathrm{P})$, followed by a nucleophilic attack of ammonia in this intermediate releasing phosphate and forming glutamine. $(5,6,7,8)$ It is found in all organisms, including Leishmania sp, presenting three types: GS-I, found in most prokaryotes, GS-II, found in eukaryotes, and GS-III, found in some prokaryotes..$^{(9)}$ The GS types I and II are dodecamers formed by two hexameric rings maintained mainly by hydrophobic interactions. The GS type III is formed by two hexameric rings associate across opposite interfaces, each ring has flipped $180^{\circ}$ with respect to its position in the other two types. ${ }^{(10,11)}$ Glutamine synthesis sequence of L. braziliensis (LbGS) is formed by two pentameric rings interacting, probably, by hydrophobic interactions due to the conservation (in relation to HsGS) of the sequence rich in prolines and lysines. Hydrogen bonds and salt bridges sustain the interaction of monomers, being interface weaker in LbGS than HsGS. ${ }^{(12)}$

\section{MATERIALS AND METHODS}

In this work, the nucleotide sequence encoding (GenBank CAM36993.1) the putative LbGS was cloned into a pET28a plasmid vector. A $42.35 \mathrm{kDa}$ protein was obtained by overexpressing LbGS in the Escherichia coli (DE3) NiCo strain with $1 \mathrm{mM}$ IPTG at $30^{\circ} \mathrm{C}$ for four hours. The recombinant protein was purified from the soluble fraction of cellular lysate using a HisTrap column in the Akta Purifier system (GE Healthcare) using buf- 
fer A (Sodium phosphate buffer pH $7.410 \mathrm{mM}, 500 \mathrm{mM}$ $\mathrm{NaCl}, 40 \mathrm{mM}$ imidazole) to equilibrate the column and a linear gradient of buffer B (pH $7.410 \mathrm{mM}$ sodium phosphate buffer, $500 \mathrm{mM} \mathrm{NaCl}, 1 \mathrm{M}$ imidazole) for elution.

We performed crosslinking experiments (XL) using the purified protein as previously described. ${ }^{(13)}$ The protein was digested with trypsin in the proportion of $1 / 50(\mathrm{E} / \mathrm{S})$ for 20 hours and the enzymatic reaction and was stopped by adding trifluoroacetic $(0.4 \% \mathrm{v} / \mathrm{v}$ final). Subsequently, the peptides were quantified using the fluorometric test - Qubit 4.0 ${ }^{\circledR}$ (Invitrogen) according to the manufacturer's recommendations. Each sample was desalted and concentrated using Stage-Tips (STop and Go-Extraction TIPs) according to literature. ${ }^{(14)}$ The peptide mixture was suspended in $0.1 \%$ formic acid and analysed as follows. An Ultimate 3000 (Thermo Fish$\mathrm{er}^{\mathrm{B}}$ ) coupled online with a Fusion Lumos Orbitrap mass spectrometer (Thermo Fisher ${ }^{\circledR}$ ) was used for generating the mass spectra data. The peptide mixture was chromatographically separated on a column $(15 \mathrm{~cm}$ in length with a $75 \mu \mathrm{m}$ I.D.) packed in-house with ReproSil-Pur C18-AQ $3 \mu \mathrm{m}$ resin (Dr Maisch GmbH HPLC) with a flow of $250 \mathrm{~nL} / \mathrm{min}$ from $5 \%$ to $50 \% \mathrm{ACN}$ in $0.1 \%$ formic acid in a 140 min gradient. The Fusion Lumos Orbitrap was set to the data-dependent acquisition (DDA) mode to automatically switch between full-scan MS and MS/MS acquisition with 60s dynamic exclusion. Survey scans $(200-1500 \mathrm{~m} / \mathrm{z})$ were acquired in the Orbitrap system with a resolution of 120,000 at $\mathrm{m} / \mathrm{z} 200$. The most intense ions captured in a 2 s cycle time were selected, excluding those unassigned and in a $1+$ charge state, sequentially isolated and HCD (Higher-energy collisional dissociation) fragmented using a normalised collision energy of 30 . The fragment ions were analysed with a resolution of 30,000 at $200 \mathrm{~m} / \mathrm{z}$. The general mass spectrometric conditions were as follows: $2.5 \mathrm{kV}$ spray voltage, no sheath or auxiliary gas flow, heated capillary temperature of $250^{\circ} \mathrm{C}$, predictive automatic gain control (AGC) enabled, and an S-lens RF level of $40 \%$. Mass spectrometer scan functions and $\mathrm{nLC}$ solvent gradients were controlled by the Xcalibur 4.1 data system (Thermo Fisher ${ }^{\circledR}$ ). Protein identification was performed using Pattern Lab for proteomics V available at http://www. patternlabforproteomics.org and a database containing 8,084 sequences of $L$. braziliensis downloaded from Uniprot. Results were filtered as described in the software's bioinformatics protocol ${ }^{(15)}$ and only the protein of interest was identified, thus achieving 0\% FDR. XL identification was performed with the Spectrum Identification Machine for Cross-Linked Peptides (SIM-XL) software that is freely available at http://www.patternlabforproteomics.org/sim-xl. ${ }^{(16)}$ The LbGS sequence from $L$. braziliensis was downloaded on March 29th, 2021, from the NCBI. The search parameters considered: fully tryptic peptide candidates with masses between 600 and 4800 Da, $20 \mathrm{ppm}$ for precursor and fragment mass. The modifications were carbamidomethylation of cysteine and oxidation of methionine as fixed and variable, respectively. The files are available in proteomics.fiocruz.br/LbGS (Supplementary data). The distance of $11.4 \AA$ between
A
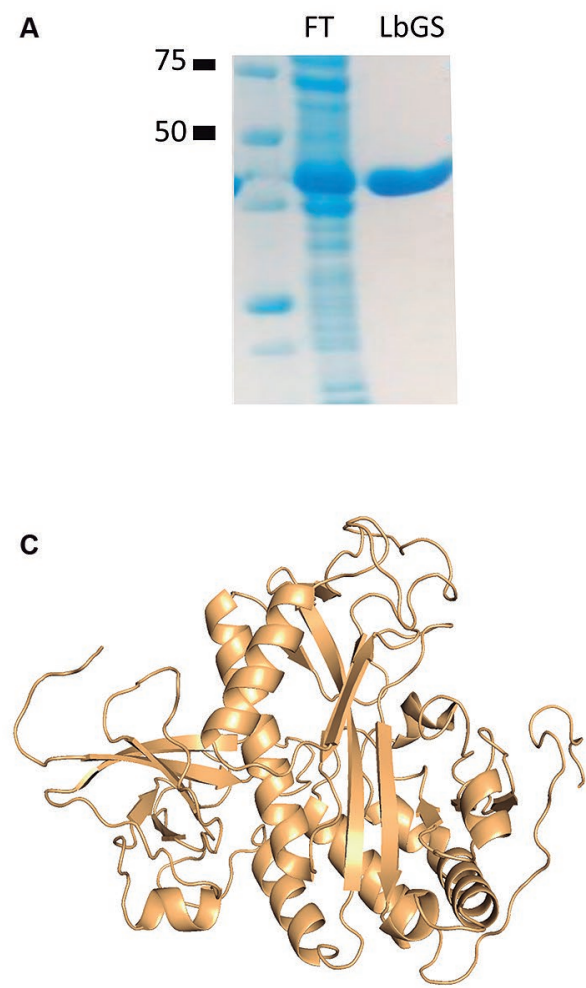

B

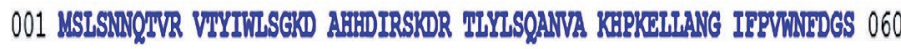 061 STGQAKGLD EILLXPYAR PCCLPRPSSK IPWLLVLAC YLPSGEPTSD NSRAAARATE 120

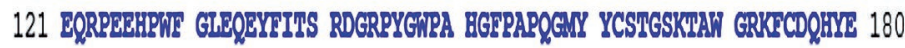 181 VCLONGLNIS GTNAEVTPGQ WETOVGPCEG LNVGDOLTVA RTVLLPLLRE EGLDADYHAR 240

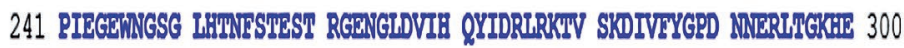 301 ISKVSEISAG VGIRCISIRI ENAVASEGKG WMEDRRPAGD ADPYLVISR RASCIGLETP 360 361 SLDLVSPTHE KDNMRSVLK

D

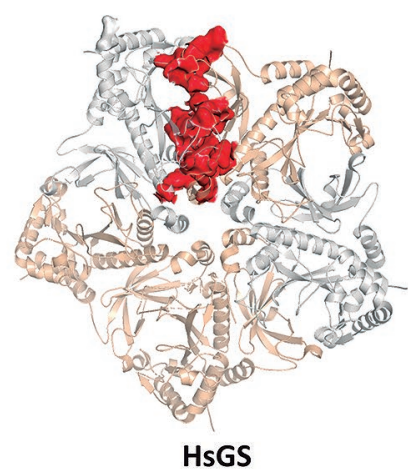

Fig. 1: (A) purified recombinant glutamine synthetase (LbGS) $(0.4 \mu \mathrm{g} / \mu \mathrm{L})$. FT indicates the flow through of chromatography. (B) LbGS sequence with peptides identified by mass spectrometry in blue. (C) the tertiary model obtained with XLMS. (D) pentameric rings from HsGS (PDB ID 2OJW) and LbGS. In red the interacting interface residues. 
cross-linked lysines identified using SIM-XL (scores limit of 1.5 for intralinks and 2.0 for interlink) ${ }^{(16)}$ was used as an input for I-TASSER. ${ }^{(17)}$ Structural analysis and visual inspection were conducted with EBI-PISA, ${ }^{(18)}$ Pymol (The PyMOL Molecular Graphics System, Version 2.0 Schrödinger), Wincoot, ${ }^{(19)}$ and ChimeraX. ${ }^{(20)}$

\section{RESULTS AND DISCUSSION}

The identity of purified protein (Fig. 1A) was confirmed by mass spectrometry (Fig. 1B). The experimental constraints obtained by XLMS are listed in Table I. The tertiary model (Fig. 1C) displayed a C-score value of 0.89 and a TM-score of $0.83 \pm 0.08$, which indicates good confidence and correct fold (TM-score $>0.5$ suggests a correct fold). ${ }^{(17,21)} 37$ out of $42 \mathrm{XL}$ distances could be placed in the monomeric model, with acceptable distances between $11.4 \AA$ and $35 \AA$ (Table I). ${ }^{(22)}$

All GS are oligomers and eucaryotes GS type II are decamers composed of pentameric rings superimposed with monomers comprised of $\sim 350$ to 420 residues. GSs have ten active sites per oligomer placed in the interface of two interacting monomers. ${ }^{(5,23)}$ The 5 XL restraints that cannot be justified by a monomeric model should indicate the region of dimerization; these results corroborate that, like other GS, the dimerization occurs from the $\mathrm{C}$-terminal region of one monomer and $\mathrm{N}$-terminal of the other (Table I).

The GS decamers present two main interaction interfaces: intra-ring forming the pentamers rings and tail-totail between superimposed pentameric rings. The human GS intra-ring interface is formed by the interaction of the $\mathrm{N}$ - and C-terminal of two subunits. ${ }^{(24)}$ Comparing LbGS and HsGS interfaces (Fig. 1D, Tables II-III), we observe a highly conserved $\mathrm{C}$-terminal region but a divergent $\mathrm{N}$ terminal (Fig. 2A). LbGS lacks an N-terminal a-helix which diminishes the size and strength of that interface (1537.4 $\AA^{2}$ and $\Delta \mathrm{G}$ of -4.3 for LbGS and $2249.1 \AA^{2}$ and $\Delta \mathrm{G}$ of -15.4 for HsGS). The number of hydrogen bonds and salt bridges in HsGS are 37 and 16, respectively (Table II). In LbGS, the numbers are quite lower: seven hydrogen bonds and nine salt bridges (Table III). Although the interface of LbGS is less stable than HsGS, the following XLs might indicate that LbGS dimerization occurs in solution: K167-K166, S02-K240, D29-K28, C162-S163, K167-S166, D314-S317, D234-K240. Regarding the tailto-tail interface, XL residues S163-K167 and S166-K167 can indicate the presence of this interface in our sample.

Studies have shown that the interaction of the pentameric HsGS model also depends on residues L139 to P160, which form a loop rich in proline and glycine, favoring hydrophobic interactions within pentamers. ${ }^{(24)}$ This loop (I138 to M159, LbGS numbering) is conserved in the LbGS structure and is also rich in prolines and glycines (Fig. 2B). However, the differences in protein sequence result in an $\beta$-sheet (R143, R144 and P145, LbGS numbering) not conserved in the homolog HsGS (Fig. 2B).

The active site of GSs comprises three regions: one for glutamate, one for ATP and one for ammonia, with very conserved residues of two subunits of the pentameric ring. ${ }^{(23)}$ Each monomer is divided into two domains, each contributing with the active site of the adjacent
TABLE I

XL restrains obtained by XLMS and spatial distance between $\mathrm{Ca}$ in LbGS model

\begin{tabular}{|c|c|c|}
\hline Residue $1(\mathrm{C} \alpha)$ & Residue $2(\mathrm{C} \alpha)$ & Distance $(\AA)$ \\
\hline $35 / \mathrm{CA}$ & $41 / \mathrm{CA}$ & 17,4 \\
\hline $298 / \mathrm{CA}$ & $308 / \mathrm{CA}$ & 13,3 \\
\hline $302 / \mathrm{CA}$ & $282 / \mathrm{CA}$ & 14,7 \\
\hline $19 / \mathrm{CA}$ & $298 / \mathrm{CA}$ & 16,6 \\
\hline $282 / \mathrm{CA}$ & $298 / \mathrm{CA}$ & 16,4 \\
\hline $282 / \mathrm{CA}$ & $317 / \mathrm{CA}$ & 14 \\
\hline 19/CA & $317 / \mathrm{CA}$ & 20,6 \\
\hline $305 / \mathrm{CA}$ & 298/CA & 13,4 \\
\hline $282 / \mathrm{CA}$ & $302 / \mathrm{CA}$ & 14,7 \\
\hline $298 / \mathrm{CA}$ & $278 / \mathrm{CA}$ & 18,9 \\
\hline $348 / \mathrm{CA}$ & 298/CA & 19,2 \\
\hline 41/CA & 298/CA & 32,5 \\
\hline $329 / \mathrm{CA}$ & $317 / \mathrm{CA}$ & 18,8 \\
\hline 303/CA & $278 / \mathrm{CA}$ & 15,1 \\
\hline 19/CA & $329 / \mathrm{CA}$ & 29,7 \\
\hline $282 / \mathrm{CA}$ & 303/CA & 14,6 \\
\hline $329 / \mathrm{CA}$ & $298 / \mathrm{CA}$ & 22,6 \\
\hline $329 / \mathrm{CA}$ & 303/CA & 26,5 \\
\hline $282 / \mathrm{CA}$ & 28/CA & 34,7 \\
\hline $41 / \mathrm{CA}$ & $317 / \mathrm{CA}$ & 33,5 \\
\hline $329 / \mathrm{CA}$ & $348 / \mathrm{CA}$ & 20,9 \\
\hline 303/CA & $329 / \mathrm{CA}$ & 26,5 \\
\hline $329 / \mathrm{CA}$ & 308/CA & 20,1 \\
\hline $282 / \mathrm{CA}$ & $329 / \mathrm{CA}$ & 26,8 \\
\hline $329 / \mathrm{CA}$ & $41 / \mathrm{CA}$ & 26,6 \\
\hline $282 / \mathrm{CA}$ & $308 / \mathrm{CA}$ & 15,6 \\
\hline $240 / \mathrm{CA}$ & 259/CA & 35.8 \\
\hline $302 / \mathrm{CA}^{*}$ & $41 / \mathrm{CA}^{*}$ & $36^{*}$ \\
\hline 44/CA* & $302 / \mathrm{CA}^{*}$ & $37,2 *$ \\
\hline $240 / \mathrm{CA}^{*}$ & $2 / \mathrm{CA}^{*}$ & $40,6^{*}$ \\
\hline $19 / \mathrm{CA}^{*}$ & $326 / \mathrm{CA}^{*}$ & $35.1^{*}$ \\
\hline $302 / \mathrm{CA}^{*}$ & $303 / \mathrm{CA}^{*}$ & $3.8^{*}$ \\
\hline $303 / \mathrm{CA}^{*}$ & 298/CA* & $9 *$ \\
\hline 278/CA* & 282/CA* & $9.2 *$ \\
\hline $163 / \mathrm{CA}^{*}$ & $167 / \mathrm{CA}^{*}$ & $9,3^{*}$ \\
\hline $326 / \mathrm{CA}^{*}$ & $329 / \mathrm{CA}^{*}$ & $8.1^{*}$ \\
\hline $281 / \mathrm{CA}^{*}$ & $282 / \mathrm{CA}^{*}$ & $3.9 *$ \\
\hline $166 / \mathrm{CA}^{*}$ & $167 / \mathrm{CA}^{*}$ & $3,9 *$ \\
\hline 17/CA* & 19/CA* & $6.6^{*}$ \\
\hline $303 / \mathrm{CA}^{*}$ & $317 / \mathrm{CA}^{*}$ & $7.8^{*}$ \\
\hline $329 / \mathrm{CA}^{*}$ & $329 / \mathrm{CA}^{*}$ & $0^{*}$ \\
\hline
\end{tabular}

*: residues that cannot be explained by a monomeric model. 
TABLE II

Intra-ring interface $\mathrm{HsGS}$

\begin{tabular}{|c|c|c|c|}
\hline \multicolumn{4}{|c|}{ Hydrogen bonds } \\
\hline \# & Structure 1 & Distance $(\AA)$ & Structure 2 \\
\hline 1 & GLY 172 & 2,88 & SER 06 \\
\hline 2 & ARG 181 & 2,97 & MET 18 \\
\hline 3 & ARG 181 & 3,05 & LEU 20 \\
\hline 4 & THR 193 & 3,07 & THR 44 \\
\hline 5 & GLY 192 & 3,82 & THR 46 \\
\hline 6 & TYR 180 & 2,81 & THR 46 \\
\hline 7 & ARG 319 & 3,10 & GLY 72 \\
\hline 8 & ARG 327 & 2,64 & ASN 74 \\
\hline 9 & ARG 319 & 3,04 & SER 75 \\
\hline 10 & ARG 327 & 2,78 & ASP 76 \\
\hline 11 & ARG 327 & 3,23 & ASP 76 \\
\hline 12 & ARG 324 & 3,19 & ASP 76 \\
\hline 13 & ARG 324 & 3,16 & ASP 76 \\
\hline 14 & ARG 319 & 3,12 & ASP 76 \\
\hline 15 & ARG 181 & 3,18 & ARG 90 \\
\hline 16 & ARG 327 & 3,67 & TYR 104 \\
\hline 17 & ARG 173 & 2,90 & GLU 230 \\
\hline 18 & GLY 166 & 2,76 & GLU 230 \\
\hline 19 & ARG 173 & 2,67 & GLU 230 \\
\hline 20 & GLY 148 & 2,66 & SER 03 \\
\hline 21 & PRO 157 & 2,39 & TYR 0 \\
\hline 22 & GLY 159 & 3,25 & ARG 41 \\
\hline 23 & GLY 159 & 3,22 & ARG 41 \\
\hline 24 & TYR 162 & 2,70 & ARG 41 \\
\hline 25 & TYR 162 & 3,09 & ASP 63 \\
\hline 26 & CYS 163 & 2,77 & CYS 42 \\
\hline 27 & ALA 167 & 3,23 & SER 03 \\
\hline 28 & ASP 174 & 2,56 & LYS 11 \\
\hline 29 & GLU 177 & 2,97 & ARG 90 \\
\hline 30 & GLU 177 & 2,87 & ARG 90 \\
\hline 31 & TYR 180 & 3,39 & THR 46 \\
\hline 32 & ILE 190 & 2,73 & THR 46 \\
\hline 33 & ALA 191 & 2,85 & THR 46 \\
\hline 34 & THR 193 & 3,10 & THR 44 \\
\hline 35 & ASP 231 & 2,65 & TYR 17 \\
\hline 36 & ALA 317 & 3,00 & SER 75 \\
\hline 37 & ALA 317 & 3,07 & SER 75 \\
\hline
\end{tabular}

Salt bridges - HsGS

\begin{tabular}{lccc}
\hline$\#$ & Structure 1 & Distance $(\AA)$ & Structure 2 \\
\hline 1 & ARG 319 & 3,89 & ASP 63 \\
\hline 2 & ARG 324 & 3,65 & ASP 76 \\
\hline & & & \\
\hline
\end{tabular}

\begin{tabular}{llll}
\hline 3 & ARG 324 & 3,19 & ASP 76 \\
\hline 4 & ARG 324 & 3,16 & ASP 76 \\
\hline 5 & ARG 319 & 3,12 & ASP 76 \\
\hline 6 & ARG 324 & 3,56 & ASP 76 \\
\hline 7 & ARG 173 & 3,67 & GLU 230 \\
\hline 8 & ARG173 & 2,90 & GLU 230 \\
\hline 9 & ARG 173 & 2,67 & GLU 230 \\
\hline 10 & ARG 173 & 3,41 & GLU 230 \\
\hline 11 & ASP 174 & 3,47 & LYS 11 \\
\hline 12 & ASP 174 & 2,56 & LYS 11 \\
\hline 13 & GLU 177 & 2,97 & ARG 90 \\
\hline 14 & GLU 177 & 3,65 & ARG 90 \\
\hline 15 & GLU 177 & 3,50 & ARG 90 \\
\hline 16 & GLU 177 & 2,87 & ARG 90 \\
\hline
\end{tabular}

TABLE III

Intra-ring interface LbGS

\begin{tabular}{|c|c|c|c|}
\hline \multicolumn{4}{|c|}{ Hydrogen bonds } \\
\hline \# & Structure 1 & Distance $(\AA)$ & Structure 2 \\
\hline 1 & LYS 167 & 2,22 & SER 140 \\
\hline 2 & THR 168 & 3,78 & GLU 229 \\
\hline 3 & GLN 184 & 2,03 & SER 02 \\
\hline 4 & THR 192 & 2,10 & ASP 29 \\
\hline 5 & ARG 314 & 2,27 & ASP 58 \\
\hline 6 & CYS 162 & 2,01 & SER 27 \\
\hline 7 & SER 190 & 2,02 & THR31 \\
\hline \multicolumn{4}{|c|}{ Salt bridges } \\
\hline \# & Structure 1 & Distance $(\AA)$ & Structure 2 \\
\hline 1 & LYS 167 & 3,21 & ASP 324 \\
\hline 2 & ARG 172 & 3,50 & ASP 29 \\
\hline 3 & ARG 314 & 2,95 & ASP 58 \\
\hline 4 & ARG 314 & 3,98 & ASP 58 \\
\hline 5 & ARG 314 & 2,59 & ASP 58 \\
\hline 6 & ARG 314 & 2,26 & ASP 58 \\
\hline 7 & ARG 314 & 3,96 & ASP 58 \\
\hline 8 & ARG 314 & 3,76 & ASP 58 \\
\hline 9 & GLU 180 & 3,91 & ARG 10 \\
\hline
\end{tabular}

monomer: N-terminal (smaller) is composed of a sheet formed by six antiparallel $\beta$-strands which two take parts of the active site; $\mathrm{C}$-terminal (the larger), formed mainly by $\alpha$-helix and six $\beta$-strands formed by most of the residues that make up the active site ${ }^{(9)}$ (Fig. 3A). In the model obtained by the crosslinking method, the active site residues E133, E135, N247, G248, H252, R294, R314, E333 and R335 (Glutamate site, LbGS numbering) (Fig. 3B), G186, S256 and R319 (ATP site, LbGS numbering) (Fig. 3C) are fully conserved together with the ammonia site, which involves three residues from 
A
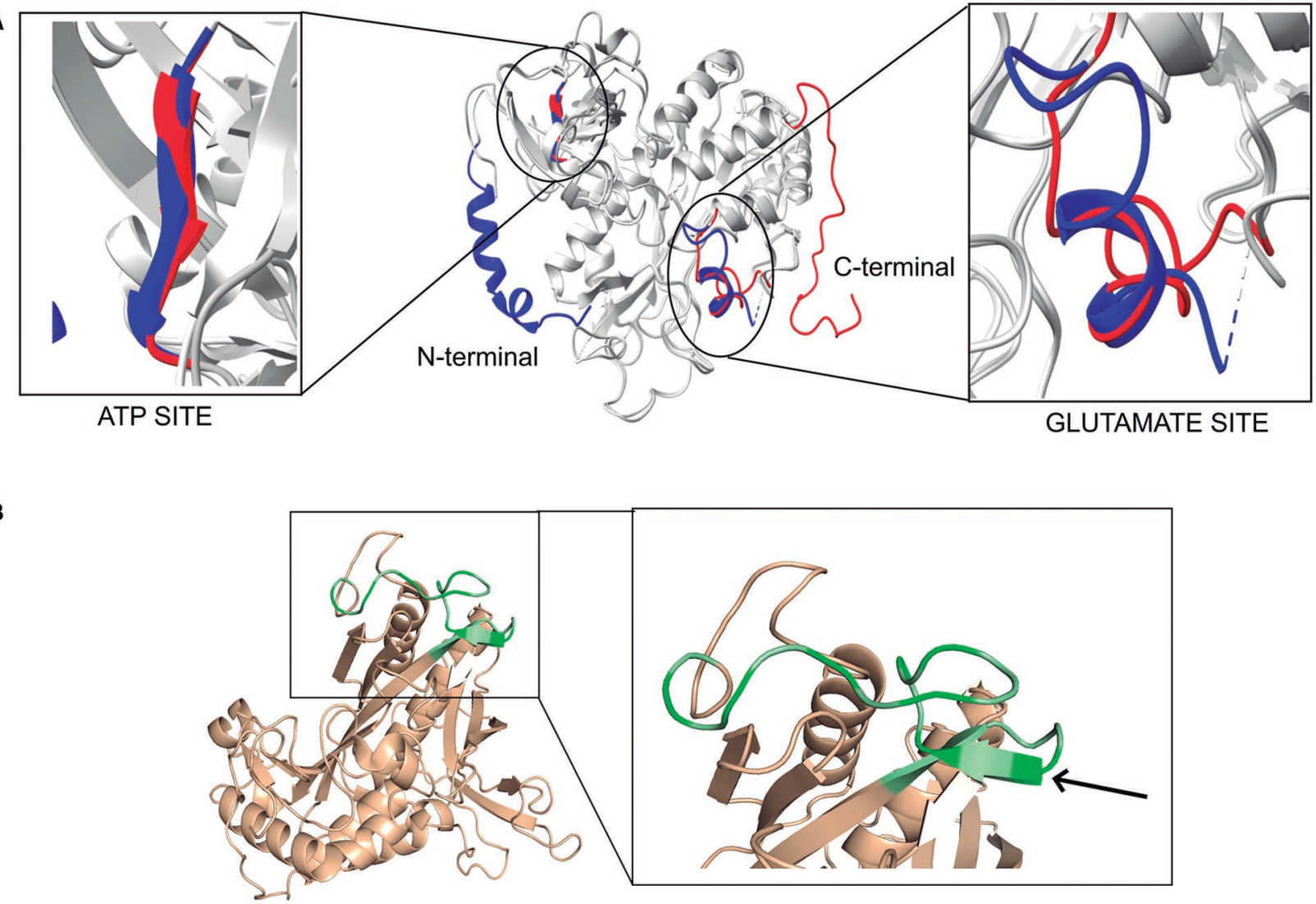

Fig. 2: (A) superposing of glutamine synthetase (LbGS) and HsGS with differences highlighted. LbGS in red, HsGS in blue. (B) LbGS monomeric model with the region from L139 to P160 highlighted (green). Dark green indicates proline and glycine residues. Arrows indicate an $\beta$-sheet not conserved in the homolog HsGS.

the C-terminal region (E195, E202 and E300, LbGS numbering) and two residues from the N-terminal region (D58 and S60, LbGS numbering) from the adjacent subunit (Fig. 3D). The structural active sites differences found by superposing the GsHS and LbGS structures reside in: (i) glutamate site from 287-303 (LbGS numbering, Fig. 2A), while R299 (PDB ID 2OJW), which is the terminal part of a loop and R294 (LbGS) which is part of an $\alpha$-helix; (ii) the ammonia site from 55-63, being D63 (PDB_ID 2OJW) parts of a $\beta$-sheet, and D58 (LbGS), part of a loop (Fig. 2A).

Finally, we also predicted a model using only the sequence of LbGS without XL restrictions. The model constructed by I-TASSER displayed good confidence scores (C-score value of -0.12 , TM-score of $0.70 \pm 0.12$ ). When both LbGS models (modeled with and without XL restrictions) are superimposed, they present an RMSD of $0.671 \AA$ and the following regions would be modeled inappropriately in the absence of the restraints obtained from experimental XL: S17-D24, N38-P53, G288-E306 (Fig. 4). Thus, it is relevant to say that spatial restrictions give modeling a sense of the real conformation of the enzyme in vitro, differently from the model generated only based on homology allowing us to evaluate their in-solution conformation, in addition to the comparison with already known 3D counterparts structures avail- able in the PDB. The technique of drug design aims both small therapeutic molecules targeting protein as itself as drug (biotherapeutics). Knowing proteins conformation in vitro and the ligand binding sites are the heart of structure-based drug development. Currently, this strategy depends not only on structural information, but also on dynamics, kinetics, and enzyme-substrate interaction data, that together provide the dynamic information on protein's in vitro conformation and flexibility and are possible due to the computational advances that emerged over years. ${ }^{(25,26)}$ Some studies have used GS enzymes as a therapeutic target to treat diseases such as cancer, malaria, and leishmaniases. ${ }^{(23,24,27,28)}$

The LbGS lacks structural and functional studies being the studies with GS from $L$. donovani (LdGS) the closest to LbGS. Kumar et al performed biochemical studies that demonstrate the enzyme's dependence on divalent metals for its optimal activity and optimum pHs from 7 to 9, similar to HsGS. ${ }^{(29)}$ Also, the provide a structural comparison of LdGS and HsGS describing relevant non-conserved residues for substrate recognition (E7, L132, S190, S249 and V205, LdGS numbering) and the importance of the electropositive potential in the active sites. ${ }^{(30)}$ These differences allowed them to find specific LdGS inhibitors, that might act in LbGS as we observed that the residues are conserved in LbGS. The 


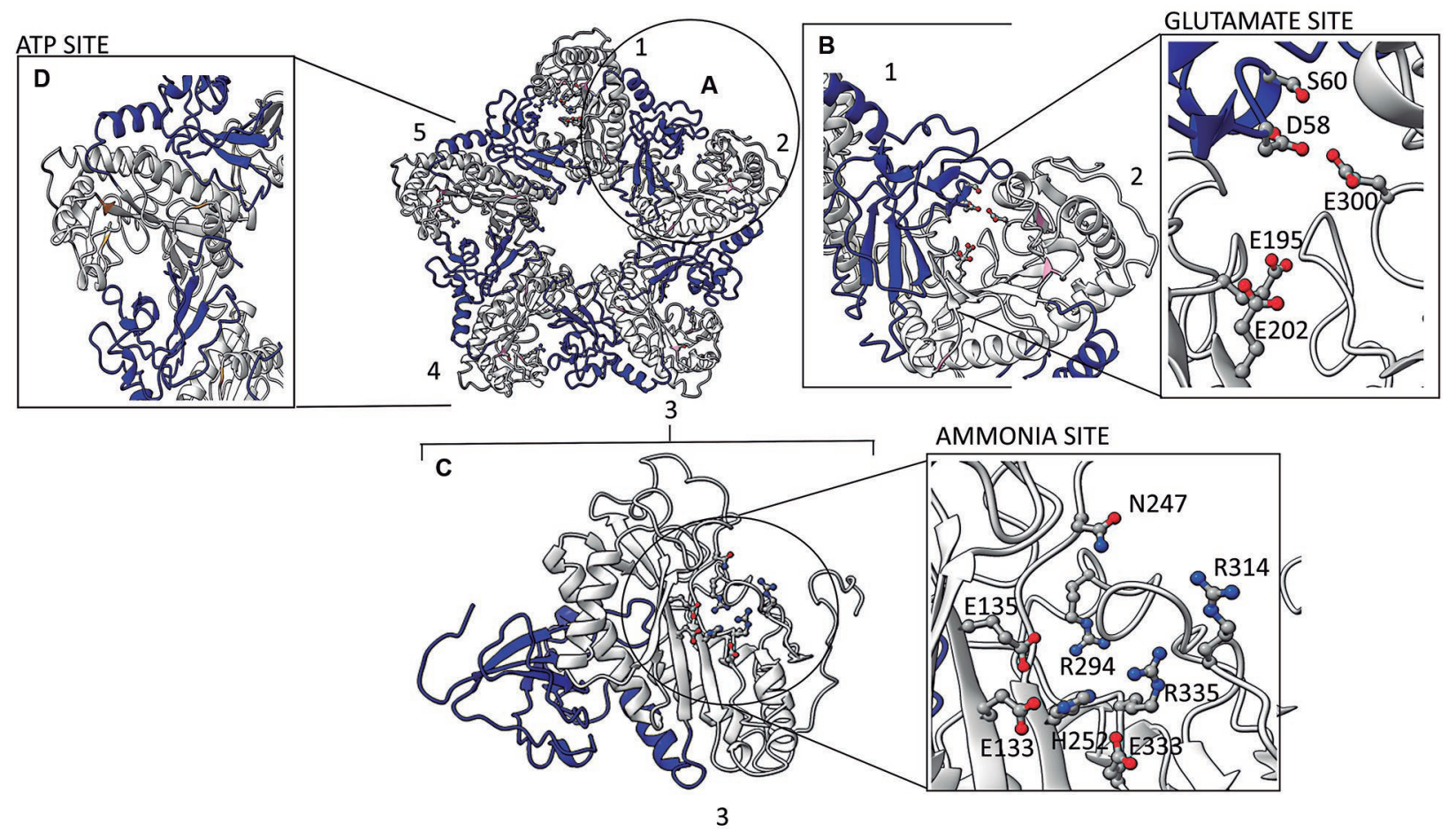

Fig. 3: the active site of glutamine synthetase (LbGS). (A) in pentamer, each monomer is divided into two domains N-terminal (blue) and Cterminal (blue). (B) the glutamate site. (C) the ammonia site. (D) ATP site. Numbers references subunits of the pentameric ring.

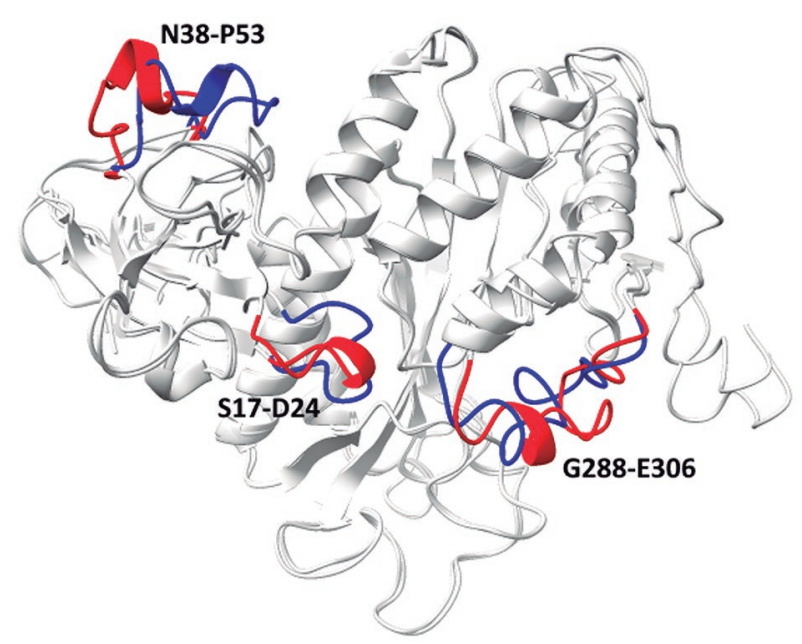

Fig. 4: superposing of glutamine synthetase (LbGS) models generated with and without XL restrictions. The divergent regions S17-D24, N38-P53, G288-E306 from LbGS modeled with (blue) and without (red) restrictions are highlighted.

potential of GS from Leishmania sp as therapeutic target was also evidenced by knock-out experiments indicating the dependence of parasite proliferation and infectivity on external supply of glutamine. . $^{(31)}$

Herein, we provide LbGS structural investigation identifying the active site, important interfaces, and unique structural features from LbGS. All these information allow investigation for new drugs.

\section{AUTHORS' CONTRIBUTION}

JYL - Experiments, data analysis, writing and review; MDMS - XLMS experiments, data analysis and review; MTM - protein production and review; PC and LC - MS/MS data analysis and review; TACBS - project coordination, writing, review and editing.

\section{REFERENCES}

1. CDC - Centers for Disease Control and Prevention. Parasites Leishmaniasis [Internet]. Centers for Disease Control and Prevention. 2020. Available from: https://www.who.int/news-room/factsheets/detail/leishmaniasis.

2. WHO - World Health Organization. Leishmaniasis [Internet]. World Health Organization. 2020. Available from: https://www. who.int/news-room/fact-sheets/detail/leishmaniasis.

3. MS/SVS - Ministério da Saúde/Secretaria de Vigilância em Saúde. Doenças tropicais negligenciadas: 30 de janeiro - Dia mundial de combate às doenças tropicais negligenciadas. Boletim Epidemiológico. Número especial. 2021. Available from: https://www. gov.br/saude/pt-br/media/pdf/2021/marco/3/boletim_especial_ doencas_negligenciadas.pdf.

4. Anversa L, Tiburcio MGS, Richini-Pereira VB, Ramirez LE. Human leishmaniasis in Brazil: a general review. Rev Assoc Med Bras. 2018; 64: 281-9.

5. Eisenberg D, Gill HS, Pfluegl GM, Rotstein SH. Structure-function relationships of glutamine synthetases. Biochim Biophys Acta. 2000; 1477: 122-45.

6. Wedler FC, Boyer PD. Substrate binding and reaction intermediates of glutamine synthetase (Escherichia coli $\mathrm{W}$ ) as studied by isotope exchanges. J Biol Chem. 1972; 247: 984-92. 
7. Wedler FC, Horn BR. Catalytic mechanisms of glutamine synthetase enzymes. Studies with analogs of possible intermediates and transition states. J Biol Chem. 1976; 251: 7530-8.

8. Woolfolk CA, Stadtman ER. Regulation of glutamine synthetase. IV. Reversible dissociation and inactivation of glutamine synthetase from Escherichia coli by the concerted action of EDTA and urea. Arch Biochem Biophys. 1967; 122: 174-89.

9. Llorca O, Betti M, González JM, Valencia A, Márquez AJ, Valpuesta JM. The three-dimensional structure of an eukaryotic glutamine synthetase: functional implications of its oligomeric structure. J Struct Biol. 2006; 156: 469-79.

10. Krajewski WW, Collins R, Holmberg-Schiavone L, Jones TA, Karlberg T, Mowbray SL. Crystal structures of mammalian glutamine synthetases illustrate substrate-induced conformational changes and provide opportunities for drug and herbicide design. J Mol Biol. 2008; 375: 217-28.

11. van Rooyen JM, Abratt VR, Belrhali H, Sewell T. Crystal structure of type III glutamine synthetase: surprising reversal of the inter-ring interface. Structure. 2011; 19: 471-83.

12. Moreira C, Ramos M, Fernandes P. Glutamine synthetase drugability beyond its active site: exploring oligomerization interfaces and pockets. Molecules. 2016; 21: 1028.

13. Pereira MBM, Santos AM, Gonçalves DC, Cardoso AC, Consonni SR, Gozzo FC, et al. $\alpha$ B-crystallin interacts with and prevents stress-activated proteolysis of focal adhesion kinase by calpain in cardiomyocytes. Nat Commun. 2014; 5: 5159 .

14. Rappsilber J, Ishihama Y, Mann M. Stop and go extraction tips for matrix-assisted laser desorption/ionization, nanoelectrospray, and LC/MS sample pretreatment in proteomics. Anal Chem. 2003; 75: 663-70.

15. Carvalho PC, Lima DB, Leprevost FV, Santos MDM, Fischer JSG, Aquino PF, et al. Integrated analysis of shotgun proteomic data with PatternLab for proteomics 4.0. Nat Protoc. 2016; 11: 102-17.

16. Lima DB, de Lima TB, Balbuena TS, Neves-Ferreira AGC, Barbosa VC, Gozzo FC, et al. SIM-XL: a powerful and user-friendly tool for peptide cross-linking analysis. J Proteomics. 2015; 129: $51-5$.

17. Yang J, Zhang Y. I-TASSER server: new development for protein structure and function predictions. Nucleic Acids Res. 2015; 43: W174-181.

18. Krissinel E, Henrick K. Inference of macromolecular assemblies from crystalline state. J Mol Biol. 2007; 372: 774-97.
19. Emsley P, Cowtan K. Coot: model-building tools for molecular graphics. Acta Crystallogr D Biol Crystallogr. 2004; 60: 2126-32.

20. Pettersen EF, Goddard TD, Huang CC, Meng EC, Couch GS, Croll TI, et al. UCSF ChimeraX: structure visualization for researchers, educators, and developers. Protein Sci. 2021; 30: 70-82.

21. Zhang Y, Skolnick J. Scoring function for automated assessment of protein structure template quality. Proteins. 2004; 57: 702-10.

22. Merkley ED, Cort JR, Adkins JN. Cross-linking and mass spectrometry methodologies to facilitate structural biology: finding a path through the maze. J Struct Funct Genomics. 2013; 14: 77-90.

23. Krajewski WW, Collins R, Holmberg-Schiavone L, Jones TA, Karlberg T, Mowbray SL. Crystal structures of mammalian glutamine synthetases illustrate substrate-induced conformational changes and provide opportunities for drug and herbicide design. J Mol Biol. 2008; 375: 217-28.

24. Moreira C, Ramos MJ, Fernandes PA. Glutamine synthetase drugability beyond its active site: exploring oligomerization interfaces and pockets. Molecules. 2016; 21(8): 1028.

25. Renaud J. Structural biology in drug discovery: methods, techniques, and practices. 1st ed. Wiley. 2020. Available from: https:// onlinelibrary.wiley.com/doi/book/10.1002/9781118681121.

26. Renaud J. The evolving role of structural biology in drug discovery. In: Renaud J, org. Structural biology in drug discovery. $1^{\text {st }} \mathrm{ed}$. Wiley. 2020. p. 1-22. Available from: https://onlinelibrary.wiley. com/doi/10.1002/9781118681121.ch1.

27. Berlicki Ł. Inhibitors of glutamine synthetase and their potential application in medicine. Mini Rev Med Chem. 2008; 8: 869-78.

28. Singh N, Siddiqi MI. Computational evaluation of glutamine synthetase as drug target against infectious diseases: molecular modeling, substrate-binding analysis, and molecular dynamics simulation studies. Med Chem Res. 2017; 26: 450-60.

29. Kumar V, Yadav S, Soumya N, Kumar R, Babu NK, Singh S. Biochemical and inhibition studies of glutamine synthetase from Leishmania donovani. Microb Pathog. 2017; 107: 164-74.

30. Kumar V, Sushma Sri N, Tripathi N, Sharma VK, Bharatam PV, Garg P, et al. Structural exploration of glutamine synthetase from Leishmania donovani: insights from in silico and in vitro analysis. Int J Biol Macromol. 2020; 146: 860-74.

31. Kumar V, Ghosh S, Roy K, Pal C, Singh S. Deletion of glutamine synthetase gene disrupts the survivability and infectivity of Leishmania donovani. Front Cell Infect Microbiol. 2021; 11: 622266. 